\title{
CELBT: An Algorithm for Efficient Cost based Load Balancing in Cloud Environment
}

\author{
Nitin Kumar Mishra \\ School of Information Technology (SOIT), \\ RGPV, BHOPAL
}

\author{
Nishchol Mishra \\ SOIT, RGPV, BHOPAL
}

\begin{abstract}
Cloud computing is interesting era of research where the multiple component, heavy hardware and architecture take part to execute the data and user processes. To maintain different virtual machines, data centers at different locations are required. Sometimes may be over burden on any datacenter and failure or downtime of the datacenter occurred. Thus in order to provide solution a load balancing technique is used which directs the request to an appropriate server for fast computation. Various traditional techniques such as Throttle, Round Robin were introduced but having some drawback with computation time and communication cost which makes them less efficient. In this paper we present CELBT (Cost Effective Load Balancing Technique) which compute the same data transfer in less computation time and cost. In this paper an evaluation is performed on CloudAnalyst tool to compute the parameters like time, cost and found the algorithm proven its effectiveness while compared with existing algorithms.
\end{abstract}

\section{Keywords}

CloudAnalyst, Load Balancing, CELBT, Virtualization.

\section{INTRODUCTION}

Cloud computing is the emerging internet based technology which emphasizes commercial computing. Cloud is a platform providing dynamic pool resources and virtualization. It also facilitates the scalable IT resources such as services, applications and infrastructure. It aims to provide the data processing in secure manner. Less effective time and communication cost is always a discussion issues while talking about the cloud computing. An efficient load balancing technique usage is the solution while dealing with multiple components.

Load sharing in cloud [1] computing environment is actually a main challenge now due to rapid evolution of internet users. To overcome this problem a distributed technique is needed [4]. Because it is not always practically possible or cost efficient to keep one or more idle services to satisfy the required demands. Jobs cannot be allocated to suitable servers and clients independently for efficient load balancing as cloud has a very complex structure and components are present throughout a wide spread area. Here some doubts are attached while jobs are allocated like how to do proper distribution of jobs among the servers available in the network so that the optimal resource utilization and a balanced traffic can be achieved in a cost efficient manner.

There are the working challenge [13] among the cloud architecture which always need to monitor and provide effect in derived algorithm.

\section{a) Energy Management:}

Energy saving is a main challenge for load balancing algorithms. Energy saving is extremely needed in cloud environment to achieve green computing. There is always a need of energy efficient algorithm which minimizes resource consumption but keeps acceptable performance [8].

\section{b) Stored Data Management:}

In the last few years exponential growth has been seen in stored data across networks. This data may be belonging to any company or any individual. The management of these data of companies or individuals, become a main challenge for cloud computing. Then how can optimum utilization of storage is achieved while maintaining fast access?

\section{c) Emergence of Small Data Centers for Cloud Computing:}

Small data centers have some benefits over large data centers [14] like they are more beneficial, cheaper and less energy consumer. Small providers can deliver cloud computing services leading to geo-diversity computing. Adequate response time with an optimal distribution of resources is a big problem in load balancing.

\section{PROBLEM DEFINITION}

The existing algorithm which considered in this research paper are Round Robin [12] and Throttle for the load balancing in cloud computing. These existing algorithms have various problems and challenges like Round Robin always required a concurrent time and thus it take heavy process in computation which affect the cost also. RR also does not guarantee for the cost effective, power saving mechanism and any fault tolerance mechanism to work on load balancing scenario whereas Throttle does not guarantee to compute high throughput and generate overhead in communication thus the response time and overall mechanism is not suitable for adoption. Throttle algorithm also suffers with its complex architecture having a large algorithm which causes a high computation cost while dealing with this load balancing technique. To overcome these challenges present in existing techniques, an efficient CELBT is proposed in this paper for better load balancing effectiveness in cloud computing applications.

\section{PROPOSED ALGORITHM}

In order to provide efficient load balancing among the cloud environment this paper presents an algorithm which is CELBT. The CELBT load balancing is a non-pre-emptive discipline, in which clustering of cloudlets and virtual machines is done on some criteria like cloudlets size and available resources of virtual machine. And then cloudlets are mapped to appropriate virtual machine for their execution which prevents unbalancing of cloud load and cloudlet lost. This algorithm is mainly focused on the proper distribution of cloudlets among the available virtual machines in such a way maximum performance can be achieved. This algorithm basically works in four steps. In first step the creation of virtual machine and cloudlets are perform and second it finds the virtual machine for the deployment. In third step cloudlets 
distribution is perform in a proposed manner. And in case of ambiguity of virtual machine when all having same number of cloudlets randomly assign one of them for Cloudlets if the storage capacity is available otherwise create new virtual machine from the container. The flow diagram for the proposed CELBT can be drawn as below:

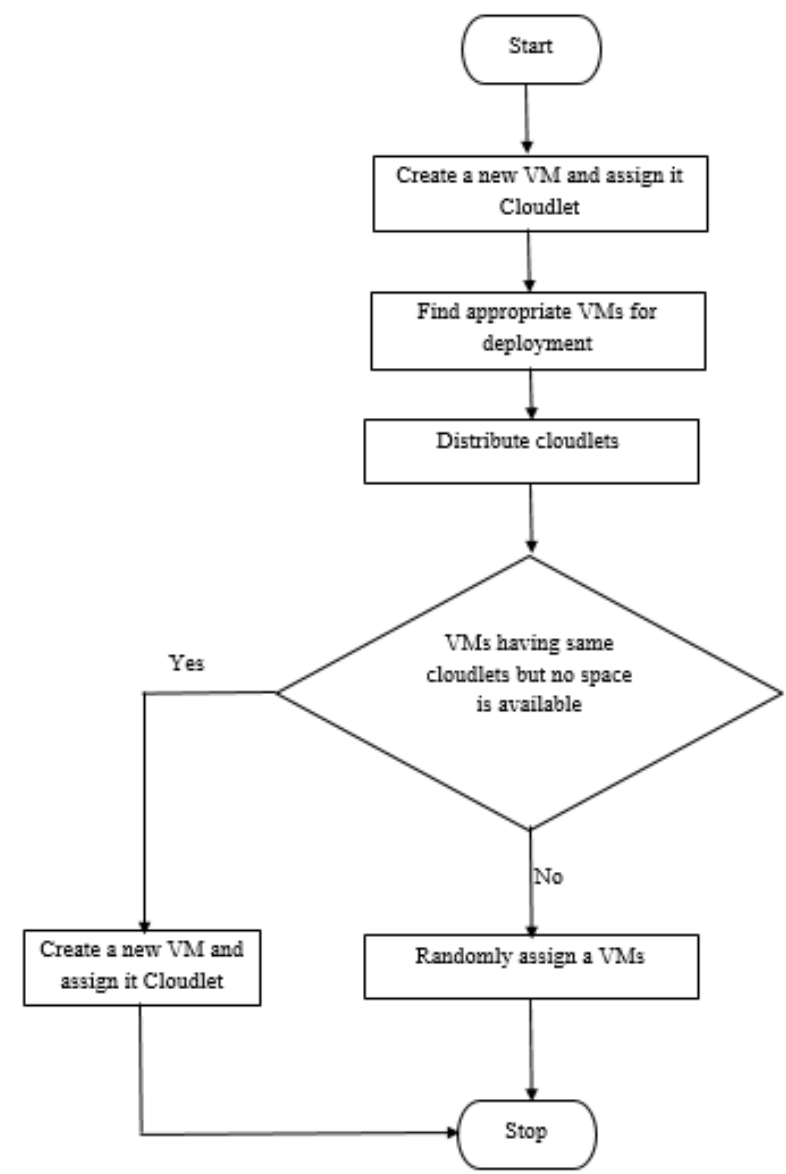

The algorithmic form of the proposed CELBT for cloud computing is shown below where input parameters for the CELBT algorithm is $\mathrm{Vm}, \mathrm{CLi}$ and $\mathrm{Ubi}$ represents virtual machine, cloudlet and user base respectively. Output parameters of the algorithm is total cost, overall response time and virtual machine statistics which is shown as output of CloudAnalyst [3] simulator tool.

\section{CELBT Algorithm for Cloud Computing}

\section{CELBT Algorithm}

Input: $\mathrm{Vm}, \mathrm{CLi}$, Ubi.

Output:TCost, overall response time, VMstatistics.

\section{[Start]}

Start Simulation;

Create Vm n, Cloudlet n, create Datacenter DCi, create Broker BRi;

for (service request end)

\{

Loadbalancing CELBT algorithm

\{ If (VMi> remaining storage)
$\{$ if ( VMi contains least cloudlet)

\{

Create VMnew $=\mathrm{VMi}$;

Allocate $\mathrm{CLi}=$ Cloudlet

If $(\mathrm{CLi}==$ remaining storage $\mathrm{VMi})$

\{

Allocate CLi having remaining storage;

\}else

\section{\{}

Distribute equal size cloudlet;

\} else

\{

Distribute dissimilar size cloudlets;

\}\}

Deployed $=$ true;

If (true)

\{

Return success;

Return parameter calculation;

Terminate;

\}else

\{

Return failure;

Stop simulation;

Terminate;

\}\}\}

[End]

\section{RESULT EVALUATION}

In order to prove that proposed CELBT algorithm performed better than others, an experimental setup is done using OS windows 10, Java platform and using Cloudsim API and the CloudAnalyst simulation tool for the experiment purpose. We have setup different $\mathrm{Vm}$, different Cloudlets, different number of brokers and implemented the algorithms Throttle, Round Robin and our proposed CELBT algorithm for monitoring the effectiveness in load balancing technique. We have taken two major computational parameter such as computation time to process the request and communication cost to process the request to prove the work performed as best. The observed results are discussed below in detail on different Vm(virtual machine) condition environment on cloud computing. These time and cost results are obtained by using CloudAnalyst simulation tool.

In table 1 results shows that proposed algorithm is taking less time with respect to RR and Throttle with varying number of virtual machines (VM25, VM50, VM75, VM100 represents $25,50,75,100$ virtual machines respectively) in CloudAnalyst simulation environment. 
Table 1: Comparison among Algorithm

\begin{tabular}{|c|c|c|c|c|c|c|}
\hline $\begin{array}{l}\text { VIRTUAL } \\
\text { MACHINE }\end{array}$ & $\begin{array}{c}\text { CELBT } \\
\text { TIME } \\
(\mathrm{ms})\end{array}$ & $\begin{array}{c}\text { CELBT } \\
\text { COMPUTATI- } \\
\text { ONAL COST } \\
\text { (\$) }\end{array}$ & $\begin{array}{c}\text { ROUND } \\
\text { ROBIN } \\
\text { TIME } \\
(\mathrm{ms})\end{array}$ & $\begin{array}{c}\text { RR } \\
\text { COMPUTATI- } \\
\text { ONAL COST (\$) }\end{array}$ & $\begin{array}{l}\text { THROTTLE } \\
\text { TIME (ms) }\end{array}$ & $\begin{array}{c}\text { THROTTLE } \\
\text { COMPUTATI- } \\
\text { ONAL COST }(\$)\end{array}$ \\
\hline VM25 & 75.30 & 11.43 & 75.34 & 13.43 & 75.39 & 13.43 \\
\hline VM50 & 118.47 & 21.46 & 118.63 & 25.98 & 118.67 & 25.98 \\
\hline VM75 & 292.21 & 31.51 & 293.47 & 38.60 & 293.35 & 38.52 \\
\hline VM100 & 293.81 & 51.07 & 293.83 & 51.07 & 294.12 & 51.07 \\
\hline
\end{tabular}

\subsection{Graphical Time Based Comparison}

In the figure 2 shows the graph among proposed, RR and throttle load balancing algorithm with respect to time for varying number of virtual machines. In this graph $\mathrm{X}$-axis represent the number of virtual machines used at a time and Y-axis used the time taken by algorithms in mili seconds.

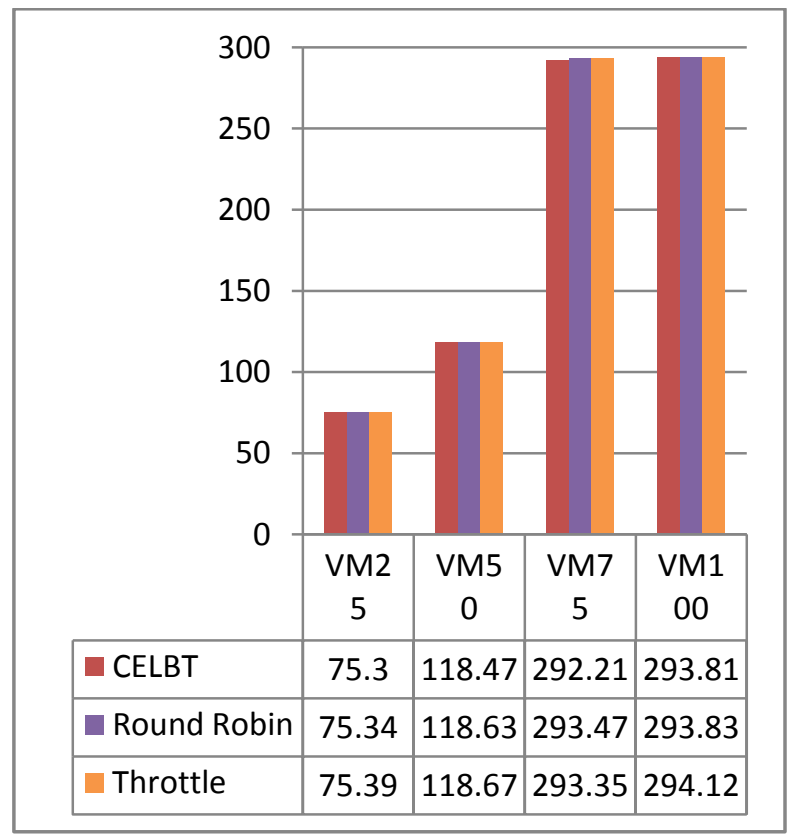

Fig 2: Time Based Comparison among LB Algorithms

\subsection{Graphical Cost Based Comparison}

Figure 3 shows the graph among algorithms with respect to cost for various number of virtual machines. In this graph $\mathrm{X}$ axis represents number of virtual machines used at any time instance and $\mathrm{Y}$-axis repesents the cost in \$.

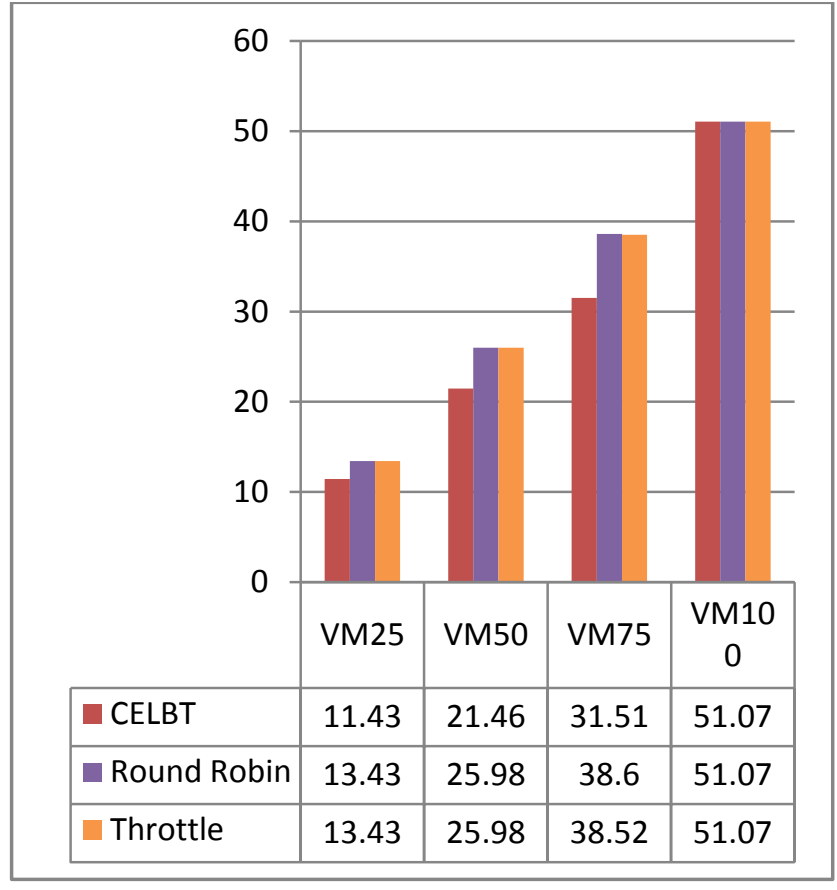

Fig 3: Cost Based Comparison of among LB Algorithms

Thus upon discussion with the statically and graphical analysis with the three algorithm including CELBT we have observed the result and found the effectiveness of our algorithm as compare with two existing algorithm which proves the derived algorithm compute better than the existing algorithm in taken parameters for computation.

\section{CONCLUSION \& FUTURE WORK}

Cloud computing is a vast area of discussion where a large data is transforming today in IT industry. This paper described the algorithm which are in trend to manipulate user request in desire server as per the server present condition and provide efficient load balancing technique which result in less time computation and thus effect in cost model also. This paper also described proposed algorithm CELBT which computes the efficient result in two parameters which discussed above as computation time and communication cost. Thus the result observed were more accurate and observed using Cloudsim simulation environment framework and analyze statically and graphical manner which proves our algorithm as a 
comparative good enough than existing cloud load balancing approaches available in cloud computing. In future work we will further going to apply the simulation work with real time scenario such as amazon and other cloud provider services with real time data to monitor the effectiveness of our algorithm.

\section{AKNOWLEDGEMENT}

This research is supported by School of Information Technology (SOIT), RGPV University (State Technological Universities of Madhya Pradesh) Bhopal, India. Sincere thanks to all the faculty members who provided insight and expertise that greatly assisted the research. Thanks to Garima Tanwar [MTech] and Vikas Yadav[ MTech] for remarks that greatly enhanced the manuscript.

\section{REFERENCES}

[1] S.Sethi,A.Sahu,andS.K.Jena,"Efficient loadbalancing incloudcomputingusingfuzzylogic",IOSRJournalofEngin eering, vol. 2, no. 7, pp.65-71, 2012.

[2] S. Wang, K. Van, W. Liao, and S. Wang, "Towards a Load Balancing in a Three-level Cloud Computing Network", Proceedings of the 3rd IEEE International Conference on Computer Science and Information Technology (ICC SIT), Chengdu, China, pp.108-113, September 2010

[3] Shahrzad Aslanzadeh, Venkatesh Mahadevan, Christopher Mcdermid, "Availability and Load Balancing in Cloud Computing," International Conference on Computer and Software Modeling IPCSIT vol.14 IACSIT Press, Singapore 2011.

[4] Sreenivas Velagapudi, M.Prathap and Kemal Mohammed, "Load Balancing Techniques: Major Challenge in Cloud Computing - A Systematic Review,'IEEE, International Conference on Electronics and Communication Systems (ICECS) - Coimbatore, India (2014).

[5] T. Kokilavani and Dr. D.I. George Amalarethinam, "Load Balanced Min-Min Algorithm for Static MetaTask Scheduling in Grid Computing," International Journal of Computer Applications Volume 20- No.2, pp.0975-8887, April 2011.

[6] V. Nae, R. Prod an, and T. Fahringer, "CostEfficient Hosting and Load Balancing of Massively Multiplayer
Online Games", Proceedings of the lith IEEE/ ACM International Conference on Grid Computing (Grid), IEEE Computer Society,pp.9- 17, October 2010.

[7] Y. Fang, F. Wang, and J. Ge, "A Task Scheduling Algorithm Based on Load Balancing in Cloud Computing", Web Information Systems and Mining, Lecture Notes in Computer Science, Vol. 6318,pp.271277,2010 .

[8] Z. Nine, M. SQ, M. Azad, A. Kalam, S. Abdullah and R. M. Rahman, "fuzzy logic based dynamic load balancing in virtualized data centers", In fuzzy system (FUZZ), IEEE International conference on, pp. 1-7, 2013.

[9] Z. Zhang, and X. Zhang, "A Load Balancing Mechanism Based on Ant Colony and Complex Network Theory in Open Cloud Computing Federation", Proceedings of 2nd International Conference on Industrial Mechatronics and Automation (ICIMA), Wuhan, China,pp.240- 243, May 2010 .

[10] Ms. Nitika, Ms. Shaveta, Mr. Gaurav Raj; "Comparative Analysis of Load Balancing Algorithms in Cloud Computing," International Journal of Advanced Research in Computer Engineering \& Technology Volume 1, Issue 3, May 2012

[11] Nidhi Jain Kansal, InderveerChana, "Cloud Load Balancing Techniques: A Step Towards Green Computing", IJCSI, Vol. 9, Issue 1, January 2012.

[12] Nusrat Pasha, Dr. AmitAgarwal and Dr. Ravi Rastogi, "Round Robin Approach for VM Load Balancing Algorithm in Cloud Computing Environment" International Journal of Advanced Research in Computer Science and Software Engineering Volume 4, Issue 5, May 2014.

[13] Sreenivas Velagapudi, M.Prathap and Kemal Mohammed, "Load Balancing Techniques: Major Challenge in Cloud Computing - A Systematic Review,'IEEE, International Conference on Electronics and Communication Systems (ICECS) - Coimbatore, India (2014).

[14] Amazon Elastic compute cloud(EC2), http://www.amazon.com/gp/browse.html?node $=2015900$ 11. 